\title{
Circulating cell-free DNA: the blood biopsy in cancer management
}

\begin{abstract}
Currently, tissue biopsy, generally from the primary tumor, is used to determine molecular profiles at a single time point, before targeted therapy commences. Tissue biopsies carry some risks for patients, they are painful, costly, time-consuming, and most importantly, may not be a true representation of tumor heterogeneity. Tumor genomes are remarkably unstable and prone to clonal expansion under selection pressure, blood biopsies via circulating cell-free DNA (cfDNA) offer what tissue biopsies can't, the opportunity to take serial samples for longitudinal monitoring of tumor genomic evolution in real time. Blood biopsy-based testing will allow clinicians to ensure treatment efficacy, monitor drug resistance, metastasis and recurrence, tailoring patients to the right treatment for the right target without delay. Tumor genome sequencing on circulating cfDNA to guide treatment decisions would be the standard-of-care of new-generation cancer management. In this review, we will briefly discuss cfDNA and its advantage over tissue biopsy, challenges and future perspectives for cancer management.
\end{abstract}

Keywords: cell-free dna, cancer, blood biopsy, tissue biopsy, next-generation sequencing, personalized medicine
Volume 2 Issue 2 - 2015

Jennifer L McLarty, ' Chen-Hsiung Yeh ${ }^{2}$

'Atherotech Diagnostics Laboratory, USA

${ }^{2}$ CirculoGene Diagnostics, USA

Correspondence: Chen-Hsiung Yeh, CirculoGene Diagnostics, 2008E Longleaf Drive, Birmingham, AL 35216, USA, Tel 12052340 I28, Email tbc2130@yahoo.com

Received: February 27, 2015 | Published: March 25, 2015
Abbreviations: cfDNA, cell-free dna; NGS, next-generation sequencing

\section{Introduction}

It is estimated that 1.6 million new cases of cancer will be diagnosed, leading to over a half a million death from cancer or about 1,600 people per day, accounting for 1 out of 4 deaths in the US. ${ }^{1}$ Major advances in genetic testing of solid tumor biopsies have fundamentally changes how cancers are targeted and treated which ultimately leads to the improved survival rates. However, solid tumor sampling is invasive, sometimes unavailable, impractical for longitudinal monitoring, heterogeneous, or unable to obtain sufficient

Table I Comparison of blood biopsy and tissue biopsy

\begin{tabular}{lll}
\hline Key characteristics & Blood biopsy & Tissue biopsy \\
\hline Invasiveness & No & Yes \\
Sample Availability Throughout the Disease Process & Yes & No \\
Sample Stability When Maintained Ex Vivo & Yes & Stable when processed \\
Utility for Longitudinally Disease Monitoring & Yes & No \\
Cost & Low & High \\
Processing Time & Short & Long (involvement of tissue sectioning, staining and pathologists) \\
Rejection/Failure Rate & Low & High (due to QNS or TNI) \\
Starting Material for Multiple Testing & Suffice & Scarce
\end{tabular}

Table Abbreviations: QNS: Quantity Not Sufficient;TNI:Tumor Not Identified

\section{History of circulating cell-free DNA}

Most of the nucleic acids (DNA and RNA) in the body are located within cells, but a fair amount of extracellular nucleic acids can also be found circulating in the bloodstream. Since the first discovery tumor content; thus, tissue biopsy sampling is a major challenge in the treatment and management of cancers. ${ }^{2}$ Blood biopsy, particularly circulating cell-free DNA (cfDNA), is emerging as a non-invasive method for cancer targeted therapy by identification of specific druggable genetic alterations in bloodstream. ${ }^{3}$ Table 1 showed side-by-side comparison between blood biopsy and tissue biopsy. Advanced technology to isolate, quantify and analyze cfDNA has led to the identification of cancer-specific aberrations in circulation such as chromosomal abnormalities, gene mutations, methylation and copy number variations which share identical characteristics of tumor tissues. ${ }^{4,5}$ The clinical utility of cfDNA in the personalized management of cancer will serve as a major game changer with the overarching goal to optimize overall cancer survival rates. of cfDNA in 1948 by Mandel and Metais in the blood, researchers have found that cfDNA track and discriminate cancer patients and healthy individuals in two folds: first by its elevated concentration and second, by the presence of tumor-specific alterations. ${ }^{6}$ Tumor-specific cfDNA has been found in a wide range of cancers, as listed by not 
limited: hematological, colorectal, pancreatic, skin, head-and-neck, lung, breast, gastric, prostate, and cervix. ${ }^{4-6}$ This suggests that cfDNA is a hallmark for all cancer and may reflect a pathological process that can be used to diagnose cancer, to monitor disease progression, and to identify treatment responses. Evidence existed in terms of the origin of these cell-free circulating genetic molecules: ${ }^{1}$ they are shed into bloodstream through "active" release of newly synthesized nucleic acids; or ${ }^{2}$ they are the "passive" end-products of necrotic and/ or apoptotic cell death. ${ }^{7,8}$

\section{Extraction of circulating cell-free DNA}

Currently, although a number of methods for extraction of cfDNA are employed, the efficiency and yield are still low due to loss of starting materials during extraction, and its quantification is variable because of lack of standardization., ${ }^{9,10}$ Nevertheless, for healthy subjects, the average concentration of circulating cfDNA is found to be $10-30 \mathrm{ng} / \mathrm{mL}$ with values of cancer patients exceeding $100 \mathrm{ng} / \mathrm{mL}$ of plasma. ${ }^{11-13}$ The estimation of cfDNA contributed by tumors using multiple methods is between 0.01 to $90 \% .{ }^{12}$ Most cfDNA fragments measured between 150 to 200 base pairs in length, with a variable half-life in the circulation ranging from 15 minutes to several hours. ${ }^{13}$ The amount of cfDNA is influenced by tumor progression, turnover of tumor, tumor size, as well as clearance, degradation, and filtering by the blood and lymphatic circulation. ${ }^{13}$ The most common published extraction methods for cfDNA are the commercially available spin column extraction kits. Other reported methods of extraction include magnetic beads, phenol/chloroform extraction, and alkaline salting. The efficiency of cfDNA extraction can directly impact the outcome of mutation detection i.e., assay sensitivity (Table 2).

Table 2 Advanced technologies employed for circulating cell-free DNA in oncology

\begin{tabular}{|c|c|c|}
\hline Technologies & Sensitivity & Clinical utility \\
\hline ARMS-PCR (Amplification Refractory Mutation System) ${ }^{5}$ & $1 \%$ & Treatment decision; Resistance \\
\hline Clamping PCR 3,22 & $0.1-1 \%$ & Treatment decision; Resistance \\
\hline castPCR (Competitive Allele-Specific TaqMan PCR) ${ }^{5,10}$ & $0.1-1 \%$ & Treatment decision; Resistance \\
\hline $\begin{array}{l}\text { ICE COLD-PCR (Improved \& Complete Enrichment CO-amplification at } \\
\text { Lower Denaturation temperature })^{23}\end{array}$ & $0.1-1 \%$ & Treatment decision; Resistance \\
\hline BEAMing Digital PCR (Beads, Emulsions, Amplification and Magnetics) ${ }^{2-4}$ & $0.01 \%$ & Treatment decision; Resistance \\
\hline Droplet Digital PCR 6 & $<0.1 \%$ & Treatment decision; Resistance \\
\hline NGS (Next-Generation Sequencing) $)^{6,16,21,22}$ & $1-5 \%$ & Treatment decision; Metastasis; Resistance; Recurrence \\
\hline
\end{tabular}

\section{Utility of circulating cell-free DNA in cancer}

Circulating cfDNA-based non-invasive method can be used to detect and monitor specific and predictive biomarkers that are recommended for the proper treatment of cancer patients according to the molecular characterization of the specific cancer. For example by using qPCR, digital PCR or sequencing, KRAS and EFGR mutation status can be obtained from cfDNA in cancer patients before, during and after targeted therapy. ${ }^{14}$ The whole exome sequencing of cfDNA by next generation sequencing (NGS) can provide a global, complete and real-time picture regarding molecular status of tumor progression. ${ }^{15}$ Most importantly, tumor load could be quantitatively estimated from ultra-deep cfDNA mutation profiling. ${ }^{16}$ Methylation status present in the tumor genome can also be detected in the cfDNA fragments. ${ }^{17}$ Overall, the development of patient-centered molecular diagnostics with blood biopsy makes perfect sense, and it's promising and beneficial to patient healthcare. Table 2 summarized advanced technologies currently deployed for circulating cell-free DNA in oncology.

\section{Challenges and future perspectives}

Current cancer management for treating cancer patients with precision therapy is still challenged by the following limitations:

Tumor heterogeneity: Tumors are heterogeneous and continuously polyclonal expansion, complicating diagnosis, treatment and the assessment of acquired resistance. Furthermore, tumor tissues are limited both spatially to the region biopsied, and temporally to the state of tumor at the time of biopsy. ${ }^{18}$

Accessibility of tumor tissue: The majority of patients treated with targeted therapies ultimately develop resistance, metastasis or recurrence. Effective monitoring with serial tumor sampling is not clinically practical with current invasive tissue biopsy techniques. Most importantly, tumor tissue may not be available or has exhausted due to multiple testing. Finally, test results from poor quality and low quantity of tissue DNA may not be obtainable. In contrast, blood biopsy, specifically cfDNA, offers an easily obtainable, minimally invasive, and longitudinal solution for precision cancer management (Table1). Clinical tests employing cfDNA are inherently specific, sensitive, and are able to capture both intra- and inter-tumor heterogeneity in real time. ${ }^{16,19}$

Overcome the limitations of blood biopsy: Circulating cfDNA is highly fragmented and presented at very low concentrations; therefore, its isolation is challenging because of the requirement of large volume input, costs, and labor-intensity. Current cfDNA recovery efficiency using the standard silica membrane/bead method is extremely low, due to the unavoidable loss in steps such as binding, washing and elution. Accordingly, it is imperative to develop novel methodologies that enable "full-recovery" of both necrotic and apoptotic cell deathassociated cfDNA for the accurate quantification as well as the detection of the specific mutation(s) in the same sample, consequently, the improvement of analytical sensitivity and specificity.

New high-sensitivity technology: Detection of low-frequency mutations through periodic "blood biopsy" analysis could monitor tumor progression before the lesions are large enough to be detected by imaging. ${ }^{3}$ Analysis beyond a single mutation could also be warranted to capture tumor heterogeneity for effective treatment decision-making. ${ }^{20}$ Blood biopsies are not as spatially limited as tissue biopsies, and can display a global spectrum of mutations that occur throughout cancer development in our body. Nevertheless, the sensitivity of conventional analytical methods such as Sanger 
sequencing is not sufficient to detect low frequency variants. In this respect, advanced NGS technology could provide a cost-effective alternative for high-throughput analysis of multiple mutations with high sensitivity. In addition, PCR-based new platforms with the features of single-molecular amplification and/or selective enrichment of tumor-specific cfDNA from a dominantly normal population, have demonstrated unprecedented assay sensitivity. ${ }^{21,22}$

Larger clinical study: Although promising proof-of-concept data in small cohorts of cancer patients from various studies have been reported, larger prospective trials are needed to confirm cfDNA clinical utility. Future studies need to demonstrate whether cfDNAderived mutation profile in blood can contribute to an improved treatment outcome of cancer patients. ${ }^{23}$

\section{Conclusion}

Blood biopsy employing cfDNA can identify multiple mutant tumor genes circulating in our bloodstream at multiple time points instead of using invasive limited-time-only tissue biopsy. Blood biopsy is expected to provide clinicians with faster, cheaper, broader, less invasive ways to assess cancer patients' clinical status and response to therapy. Blood biopsy-based tests for multiple mutation profiles via NGS could become a gold standard of new-generation cancer management in the era of precision medicine.

\section{Acknowledgements}

None.

\section{Conflict of interest}

The author declares no conflict of interest.

\section{References}

1. American Cancer Society.

2. Holdhoff M, Schmidt K, Donehower R, et al. Analysis of circulating tumor DNA to confirm somatic KRAS mutations. J Natl Cancer Inst 2009;101(18):1284-1285.

3. Diaz LA, Williams RT, Wu J, et al. The molecular evolution of acquired resistance to targeted EGFR blockade in colorectal cancers. Nature 2012;486(7404):537-540.

4. Diehl F, Li M, Dressman D, et al. Detection and quantification of mutations in the plasma of patients with colorectal tumors. Proc Natl Acad Sci USA. 2005;102(45):16368-16373.

5. Allegra CJ, Jessup JM, Somerfield MR, et al. American Society of Clinical Oncology provisional clinical opinion:testing for KRAS gene mutations in patients with metastatic colorectal carcinoma to predict response to anti-epidermal growth factor receptor monoclonal antibody therapy. J Clin Oncol. 2009;27(12):2091-2096.

6. Alix-Panabieres C, Schwarzenbach H, Pantel K. Circulating tumor cells and circulating tumor DNA. Annu Rev Med. 2012;63:199-215.

7. Stroun M, Lyautey J, Lederrey C, et al. About the possible origin and mechanism of circulating DNA apoptosis and active DNA release. Clin Chim Acta. 2001;313(1-2):139-142.
8. van der Vaart M, Pretorius PJ. Circulating DNA. Its origin and fluctuation. Ann N Y Acad Sci. 2008;1137:18-26.

9. Wimberger P, Roth C, Pantel K, et al. Impact of platinum-based chemotherapy on circulating nucleic acid levels, protease activities in blood and disseminated tumor cells in bone marrow of ovarian cancer patients. Int J Cancer. 2011;128(11):2572-2580.

10. Kamat AA, Baldwin M, Urbauer D, et al. Plasma cell-free DNA in ovarian cancer: an independent prognostic biomarker. Cancer. 2010;116(8):1918-1925.

11. Allen D, Butt A, Cahill D, et al. Role of cell-free plasma DNA as a diagnostic marker for prostate cancer. Ann N Y Acad Sci. 2004;1022:7680 .

12. Schwarzenbach H, Stoehlmacher J, Pantel K, et al. Detection and monitoring of cell-free DNA in blood of patients with colorectal cancer. Ann N Y Acad Sci. 2008;1137:190-196.

13. Fleischhacker M, Schmidt B. Circulating nucleic acids (CNAs) and cancer-a survey. Biochim Biophys Acta. 2007;1775(1):181-232.

14. Bidard FC, Fehm T, Ignatiadis $\mathrm{M}$, et al. Clinical application of circulating tumor cells in breast cancer: Overview of the current interventional trials. Cancer Metastasis Rev. 2013;32(1-2):179-188.

15. Diaz LA Jr, Sausen M, Fisher GA, et al. Insights into therapeutic resistance from whole genome analyses of circulating tumor DNA. Oncotarget. 2013;4(10):1856-1857.

16. Bettegowda C, Sausen M, Leary RJ, et al. Detection of circulating tumor DNA in early- and late-stage human malignancies. Sci Transl Med. 2014;6(224):224ra24.

17. Mori T, O'Day SJ, Umetani N, et al. Predictive utility of circulating methylated DNA in serum of melanoma patients receiving biochemotherapy. J Clin Oncol. 2005;23(36):9351-9358.

18. Gerlinger M, Rowan AJ, Horswell S, et al. Intratumor heterogeneity and branched evolution revealed by multiregion sequencing. $N$ Engl $J$ Med. 2012;366(10):883-892.

19. Dawson SJ, Tsui DW, Murtaza M, et al. Analysis of circulating tumor DNA to monitor metastatic breast cancer. $N$ Engl $J$ Med. 2013;368(13):1199-1209.

20. Sequist LV, Waltman BA, Dias-Santagata D, et al. Genotypic and histological evolution of lung cancers acquiring resistance to EGFR inhibitors. Sci Transl Med. 2011;23(75):75ra26.

21. Beck J, Hennecke S, Bornemann-Kolatzki K, et al. Genome aberrations in canine mammary carcinomas and their detection in cell-free plasma DNA. PLoS One. 2013;8(9):e75485.

22. Won JK, Keam B, Koh J, et al. Concomitant ALK translocation and EGFR mutation in lung cancer: a comparison of direct sequencing and sensitive assays and the impact on responsiveness to tyrosine kinase inhibitor. Ann Oncol. 2015;26(2):348-354.

23. Milbury CA, Li J, Makrigiorgos GM. Ice-COLD-PCR enables rapid amplification and robust enrichment for low-abundance unknown DNA mutations. Nucleic Acids Res. 2011;39(1):e2. 